\title{
CONTRIBUCIÓN AL CONOCIMIENTO DE LA VEGETACIÓN DE LAS SABANAS DE CASANARE (COLOMBIA)
}

\section{CONTRIBUTION TO KNOWLEDGE OF SAVANNAS VEGETATION OF CASANARE (COLOMBIA)}

\author{
Luz Piedad Romero-Duque ${ }^{1}$, Francisco Castro-Lima ${ }^{2}$, Ángela Rentería-Mosquera ${ }^{3}$
}

${ }^{1}$ Doctora en Ciencias Biológicas, Directora Maestría en Ciencias Ambientales. Universidad de Ciencias Aplicadas y Ambientales U.D.C.A, calle 222 No. 55- 37, Bogotá, Colombia, e-mail: luz.romero@udca.edu.co; ${ }^{2}$ Ingeniero Agrónomo. Fundación Horizonte Verde, e-mail: bojonawi@gmail.com; ${ }^{3}$ Ingeniera Geógrafa y Ambiental, e-mail: alegna164@gmail.com

\author{
Rev. U.D.C.A Act. \& Div. Cient. 21(1): 197-205, Enero-Junio, 2018 \\ https://doi.org/10.31910/rudca.v21.n1.2018.678
}

\section{RESUMEN}

Se estudió la diversidad vegetal en parcelas de bosques de galería, bosques de vega, morichales, matas de monte, bancos de sabana, bajos de sabana y esteros, así como de inventarios ad libitum. En total, se registraron 318 especies y 206 géneros de 80 familias, siendo la más diversa la familia Poaceae, con 29 especies, seguida de Euphorbiaceae, con 17 especies. Los géneros más diversos fueron Phyllantus y Panicum, con siete especies. Los tipos de vegetación con mayor riqueza fueron los bosques de galería, los bajos de sabana y los morichales. La composición, a nivel de familias en los bosques, es similar a la reportada en otros estudios, pero las especies son diferentes, sugiriendo dominancia local, debida a la heterogeneidad espacial. Con este estudio, se incrementó el número de especies registradas para el departamento de Casanare, de 1.479 a 1.562.

Palabras clave: Composición botánica, sabanas tropicales, bosques tropicales, plantas acuáticas. AGROVOC multilingual agricultural Thesaurus.

\section{SUMMARY}

We studied plant diversity in forest plots of bosques de galería, bosques de vega, morichales, matas de monte, bancos de sabana, bajos de sabana y esteros, as well as ad libitum inventories. A total of 318 species and 80 genera of 206 families were found, being Poaceae the most diverse family with 29 species, followed by Euphorbiaceae with 17 species. The most diverse genera were Phyllantus and Panicum with seven species. The types of vegetation with the greatest diversity were bosques de galería, bajos de sabana and morichales. The composition of families in forests is similar to that reported in other studies, but species composition is different suggesting local dominance due to spatial heterogeneity. With this study, there was an increase in the number of species recorded for the department of Casanare from 1479 to 1562.

Key words: Botanical composition, savannas, tropical forests, aquatic plants. AGROVOC multilingual agricultural Thesaurus.

\section{INTRODUCCIÓN}

Las sabanas suramericanas corresponden a casi el $10 \%$ del total de bioma sabana tropical (269 millones de hectáreas) (Grace et al. 2006). En Colombia, corresponden a una cuarta parte del territorio nacional $\left(250.000 \mathrm{~km}^{2}\right)$, donde son objeto de una fuerte presión antrópica, especialmente, por la extracción de petróleo (Rausch, 2009), la agricultura intensiva a gran escala, el incremento de la población humana (Correa et al. 2005), la expansión de la infraestructura vial y los cultivos de uso ilícito, lo que ha impactado su estructura y funcionamiento. Romero-Ruiz et al. (2004) registraron que el 76,5\% de la Orinoquía colombiana, donde se localizan las sabanas tropicales, está representado por coberturas naturales y el resto por coberturas antrópicas. Mora-Fernández et al. (2015) señalan que el departamento de Casanare es uno de los más transformados del país (19\%) y que sus ecosistemas están entre los menos representados en el SINAP (Usma \& Trujillo, 2011). A pesar de su importancia ecológica y socioeconómica, el conocimiento sobre la diversidad florística, tanto a escala regional como local de esta parte del país, es escaso (Mora-Fernández et al. 2015). Fernández et al. (2010) señalan que de la región Orinocense, la flora más conocida es la que tiene influencia andina y la de la Macarena, mientras que, del resto de la región, se tiene un conocimiento bajo a muy bajo; dentro de los trabajos en estas zonas destacan los de Parra (2006) y Castro-Lima (2010). El primero, se concentra en las especies dominantes y la abundancia cualitativa de las formaciones vegetales el municipio de Puerto Carreño, mientras que el segundo describe la flora 
de 19 ecosistemas del Andén Orinoqués, del departamento del Vichada. También destacan los trabajos de Rudas et al. (2002), quienes proponen una zonación fisionómico-fisiográfica de la vegetación en La Ceiba, una comunidad localizada en el municipio de Puerto Inírida (Guainía) y de Cárdenas (2007), quien realiza una descripción, un análisis florístico y fitogeográfico de la flora del municipio de Inírida. Adicionalmente, destacan los trabajos de Etter (2001), quien hace una descripción de aspectos fitogeográficos del Escudo Guyanes; de Cortés et al. (1998), quienes realizan un catálogo preliminar de la flora de la sierra de Chiribiqute; de Vincelli (1981) y Barbosa (1992), con su estudio de la flora del Parque Nacional Natural El Tuparro.

Para el departamento del Casanare, se cuenta con el trabajo de Córdoba et al. (2011), quienes hicieron una revisión de literatura secundaria y levantamientos florísticos en los municipios de Tauramena, Hato Corozal y Trinidad y Orocue; sin embargo, los mismos autores señalan que, aunque los aportes científicos han sido valiosos, aún son insuficientes, debido a la alta diversidad ecosistémica de la región. Esta situación plantea la necesidad de ampliar los estudios florísticos, que per- mitan tomar decisiones sobre su conservación y manejo. Este estudio, se constituye en una contribución al conocimiento de la flora del departamento del Casanare, mediante una descripción de la vegetación de diferentes tipos de cobertura vegetal y de inventarios en recorridos adicionales.

\section{MATERIALES Y MÉTODOS}

El departamento de Casanare, se encuentra localizado entre los $4^{\circ} 17^{\prime}$ y $6^{\circ} 20^{\prime}$ ' N y los 69 $50^{\prime}$ y $73^{\circ} 04^{\prime}$ W (Domínguez, 1998) y ocupa una extensión territorial de $44.640 \mathrm{~km}^{2}(4 \%$ del territorio nacional) (Figura 1). Según la clasificación de Holdridge (1947), el departamento presenta cinco clases de clima (extremadamente frío y pluvial, muy frío y pluvial, frío y muy húmedo, medio y húmedo, y cálido y húmedo). Este estudio, se concentró en el clima cálido y húmedo, que registra una temperatura promedio anual de $26 \mathrm{C}^{\circ}$ y una precipitación media multianual de $2.236 \mathrm{~mm}$, de comportamiento unimodal, con lluvias abril y octubre, siendo marzo y noviembre los meses de transición, entre la temporada seca y la húmeda. La zona de estudio corresponde a la unidad geomorgológica llanura aluvial de inundación y de desborde, con una zona

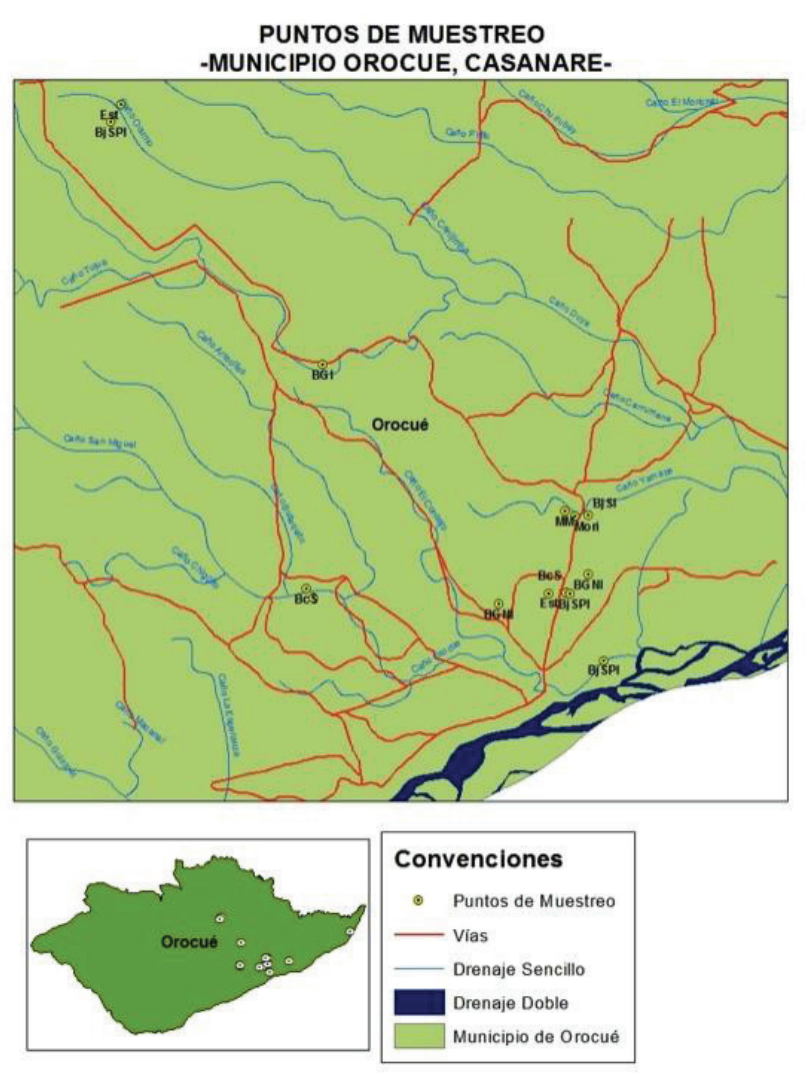

Figura 1. Localización del municipio de Orocué (Casanare, Colombia). Los puntos corresponden a los sitios de muestreo. BGI: bosque de galería inundable; BGNI: bosque de galería no inundable; BVNI: bosque de vega no inundable; MM: mata de monte; Mori: Morichal; BjI: bajo de sabana inundable; BjPI: bajo de sabana parcialmente inundable; BcS: banco de sabana y Est: estero. 
plana con pendientes de 0 a 3\% y una zona de relieve ligeramente ondulado, con pendientes de 3 a 7\%. Esta zona corresponde a planicies altas no inundables y bajas inundables (Aguirre, 1999), dónde se encuentran sabanas inundables, bosques de galería, matas de monte y morichales.

Para caracterizar la vegetación, se seleccionaron los tipos de vegetación más representativos de la región (Figura 1): 1) bosque de galería no inundable (BGNI), en las veredas Piñalito, finca Mayquetía y Caño San Miguel, Hato San Miguel; 2) bosque de galería inundable (BGI), en la vereda La Esmeralda, fincas Miraflores y El Palmar; 3) bosque de vega no inundable (BVNI) y 4) bosque de vega inundable (BVNI)n en la vereda Tapaojo, en la finca La Arenosa y Resguardo indígena Guacacías, respectivamente; 5) matas de monte (MM), según la definición de Correa et al. (2005), en la vereda La Esmeralda, finca Las Malvinas 2; 6) morichales (Mor), en la vereda La Esmeralda, finca Tijuana y 7) esteros (Est), en la vereda La Esmeralda, finca Las Malvinas y en el Pozo Tucuso de Ecopetrol.

De igual forma, se seleccionaron sabanas, siguiendo las clasificaciones de Ramia (1967) y Tejos (2000): 8) banco de sabanas (BcS - sabanas con inundación leve), en las veredas La Esmeralda, finca Las Malvinas y Caño San Miguel, Hato San Miguel; 9) bajo de sabana parcialmente inundable (BjSPI - sabanas con inundación media), en las veredas La Esmeralda, finca Tijuana y Piñalito finca Mayquetía; 10) bajo de sabana inundable (BjSI - zonas de transición sabana-estero), en la vereda La Esmeralda finca Las Malvinas y en el Pozo Tucuso de Ecopetrol. De cada tipo de vegetación, se muestrearon dos sitios, excepto para el bosque de vega inundable y la mata de monte, debido a dificultades logísticas, durante el desarrollo del estudio. En cada tipo de bosque, se estableció una parcela de $50 \times 20 \mathrm{~m}$, donde se identificaron, en lo posible hasta especie, todos los individuos que se encontra- ron dentro de la misma. A cada individuo, se le midió el DAP y se le estimó la altura. Se obtuvieron los valores de densidad, abundancia y área basal y, a partir de los dos últimos, se obtuvo el índice de valor de importancia de las especies. Los individuos, se clasificaron en cinco categorías diamétricas $(0-4,9 ; 5-9,9 ; 10-19,9 ; 20-49,9 \mathrm{y} \geq 50 \mathrm{~cm})$, para detallar la estructura del bosque y su contribución al área basal. En las sabanas y esteros, se registraron todos los individuos que estuvieron enraizados o presentes en un cuadrante de $1 \mathrm{~m}^{2}$, se determinaron hasta especie y se les registró el porcentaje de cobertura dentro del cuadrante. De igual forma, y con el fin de complementar la información, se realizaron recorridos generales, en los que se hicieron colectas botánicas adicionales ad libitum. Todos los especímenes colectados reposan en los herbarios de la Universidad de Ciencias Aplicadas y Ambientales U.D.C.A y de la Universidad de los Llanos UNILLANO. La identificación, se realizó mediante el uso de claves taxonómicas y colecciones de referencia depositados en el herbario LLANOS. Para este estudio, se tomó como referencia, el sistema de clasificación de Cronquist (1981), actualizado con APG III (2009).

\section{RESULTADOS Y DISCUSIÓN}

De manera general, se registraron un total de 318 especies (289 en parcelas, 38 en recorridos ad libitum y 9 presentes en parcelas y recorridos), 207 géneros y 81 familias (Tabla 1). La familia más diversa fue Poaceae (29 spp.), seguida de Euphorbiaceae (17 spp.), Fabaceae (16 spp.), Rubiaceae (15 spp.), Melastomataceae (14 spp.), Cyperaceae (12 spp.) y Moraceae (10 spp.). Los géneros con mayor número de especies registradas fueron Phyllantus y Panicum (7 spp. cada uno) y Miconia y Ficus (6 spp. cada uno). Los resultados del presente estudio, en el que de manera general para los ecosistemas de sabana estudiados se registra a las familias Poaceae, Fabaceae, Euphorbiaceae, Rubiaceae y Melastomataceae como las más diversas, coinciden con lo reportado

Tabla 1. Número de familias, especies e individuos, en diferentes tipos de bosque del departamento de Casanare (Colombia).

\begin{tabular}{|l|c|c|c|c|c|c|c|c|c|c|}
\hline & \multicolumn{10}{|c|}{ Tipo de Vegetación } \\
\hline & BGI & BGNI & BVI & BVNI & MM & Mori & BjSI & BjSPI & BcS & Est \\
\hline No. Familias & 36 & 37 & 26 & 22 & 22 & 24 & 19 & 23 & 17 & 9 \\
\hline No. Especies & 67 & 71 & 44 & 31 & 31 & 51 & 56 & 70 & 32 & 19 \\
\hline No. Individuos & 920 & 1068 & 256 & 295 & 205 & 475 & 179 & 274 & 127 & 111 \\
\hline
\end{tabular}

BGI: bosque de galería inundable; BGNI: bosque de galería no inundable; BVNI: bosque de vega no inundable; MM: mata de monte; Mori: Morichal; BjI: bajo de sabana inundable; BjPI: bajo de sabana parcialmente inundable; BcS: banco de sabana; Est: estero. 
por Córdoba et al. (2011) y Duno de Stefano et al. (2007), quienes encontraron que Rubiaceae, Fabaceae y Poaceae son las mejor representadas, tanto a nivel de especies como de géneros, en el departamento de Casanare. De igual forma, coincide con Córdoba et al. (2011), en que uno de los géneros más diversos es Miconia.

Los ecosistemas de bosque presentaron una alta variación en la composición y en la abundancia vegetal. Los BGNI registraron el mayor número de familias y especies (37 familias y 71 spp.) (Tabla 1). En estos bosques, Annonaceae y Chrysobalanaceae fueron las familias con mayor número de especies (6 y 5 spp., respectivamente), Arecaceae. la más abundante (705 ind.) y Attalea insignis (Mart.) Drude. la especie más abundante (641 ind.). Por su parte, las familias con mayor número de especies en los BGI fueron Apocynaceae y Clusiaceae (5 spp., cada una) (Tabla 1) y la especie con mayor número de individuos fue Protium llanorum Cuatrec. (102 ind.). Apocynaceae, también fue una de las familias más abundantes en los BVI, junto con Euphorbiaceae, Hippocrateaceae, Melastomataceae, Mimosaceae y Sapotaceae (3 spp., cada una), pero la más abundante fue Melastomataceae (42 ind.) y las especies más abundantes fueron Mouriri guianensis Aubl. y Tapura acreana (Ule) Rizzini (37 y 36 ind., respectivamente). Euphorbiaceae fue la familia más abundante y diversa en los BVNI (90 ind.; 4 spp.) y la especie más abundante Attalea butyracea (72 ind.). Por otro lado, en las MM Lauraceae fue la familia más abundante (119 ind.), mientras que Melastomataceae tuvo el mayor número de especies (5 spp.), siendo Ocotea longifolia Kunth la especie más abundante (65 ind.). Finalmente, en los Mor, la familia más abundante fue Moraceae (157 ind.), la que presentó mayor número de especies fue Melastomataceae (4 spp.) y la especie más abundante fue Maquira coriacea (155 ind.).

Los resultados de este estudio, para bosques ribereños, contrastan en composición y en abundancia vegetal con estudios reportados para ecosistemas del mismo tipo, en otras zonas de la región. Los resultados del presente estudio (171 especies, 120 géneros y 55 familias) coinciden con los de Cabrera-Amaya \& Rivera-Díaz (2016), quienes en bosques de galería, vega y morichales del río Pauto (Casanare), reportaron 185 especies, 127 géneros y 56 familias; sin embargo, contrasta la composición y la abundancia, ya que los autores reportan a Rubiaceae, Fabaceae, Moraceae y Myrtaceae, como las familias con mayor número de especies, mientras que en el presente estudio solo Moraceae se reporta como la familia con mayor número de especies en los Mor. Otro aspecto contrastante con los resultados obtenidos por Cabrera-Amaya \& Rivera-Díaz (2016), quienes reportan de 7 a 15 especies para las familias con mayor número de especies, mientras que, en el presente estudio, no superan las 6 especies, a pesar que los dos estudios muestrearon 9 parcelas de $1.000 \mathrm{~m}^{2}$ y reportan estos bosques como secundarios.
De igual forma, los resultados del presente estudio contrastan con los reportados por Veneklaas et al. (2005). El autor reporta para un bosque de galería en la cuenca del río Yacao (Meta, Colombia), 7 familias, como las que presentan un mayor número de especies (Leguminosae, Arecaceae, Rubiaceae, Chrysobalanaceae, Euphorbiaceae, Melastomataceae, Myrtaceae), pero solo comparte una con el presente estudio (Chrysobalanaceae); sin embargo, a pesar de esta diferencia, los dos trabajos comparten 46 especies, una de las cuales, también es reportada por Veneklaas et al. (2005), con un alto índice de valor de importancia, Himatanthus articulatus (Vahl) Woodson). Es importante señalar que Veneklaas et al. (2005) registraron 147 especies de árboles de $\geq 5 \mathrm{~cm}$ de DAP, en 3,2ha, mientras que, en este estudio, se registraron 98, en 0,2ha. En las MM del presente estudio, Lauraceae y Melastomataceae, presentan alta riqueza específica, al igual que lo reportado por Mendoza (2011); no obstante, estos dos estudios no comparten especies con el mayor índice de valor de importancia, lo que se puede deber a la diferencia entre las matas de monte estudiadas, ya que las de Mendoza (2011) presentaron mejor estado de conservación, debido a que se localizan en el Parque Nacional Natural El Tuparro. Por otro lado, Delascio (1990) reportó a Cyperaceae, Poaceae y Melastomataceae como las familias con mayor número de especies en los Mor, mientras que Lasso et al. (2013) reportaron a Melastomataceae y Moraceae; resultados que coinciden con lo registrado en el presente estudio, en el que Melastomataceae fue la familia con mayor número de especies. De igual forma, tanto en el presente estudio como en el de Lasso et al. (2013), Mauritia flexuosa L. f. var. venezuelana Steyerm fue la especie con mayor IVI, pero, adicionalmente, también es registrada en los trabajos de Delascio (1990) y de Aristeguieta (1968), para morichales llaneros del norte del Orinoco.

De los 3.910 individuos registrados en las parcelas de este estudio, 691 corresponden a vegetación de sabana y esteros y representaron 29 familias y 111 especies (Tabla 1). Los Est presentaron el menor número de familias y especies (Tabla 1); sin embargo, presentaron valores de porcentaje de cobertura similares a los demás tipos de vegetación de sabana, excepto los BcS, que registraron valores por encima del 70\%. Los Est presentaron el menor número de individuos y la más baja densidad (111 ind. y 5,5 ind $/ \mathrm{m}^{2}$ ). Las familias más abundantes en estos ecosistemas fueron Alismataceae (30 ind.) y Poaceae (20 ind.); sin embargo, las familias más diversas fueron Euphorbiaceae y Poaceae (4 spp. cada una). En los demás tipos de vegetación, la familia Poaceae fue la más abundante y la más diversa. En los Est, la especie más abundante fue Eleocharis filiculmis Kunth (20 ind.), mientras que, en los BcS, Axonopus purpusii (Mez) Chase (20 ind.), en los BjSI, Panicum trichoides Sw. (16 ind.) y en los BjSPI, Scleria melaleuca Rchb. ex Schltdl. \& Cham. (15 ind.). 
En los ecosistemas de sabana de este estudio, las familias dominantes fueron Poaceae, Cyperaceae y Lamiaceae, lo que coincide con las reportado por Rippstein et al. (2001) y Serna-Isaza et al. (2001), para quienes Poaceae, Fabaceae y Cyperaceae son las familias más representativas de la zona de Carimagua y de la Altillanura plana y la Serranía de los llanos orientales de Colombia, respectivamente. De igual forma, coinciden con Baruch (2005) y Sarmiento \& Vera (1979), para quienes Poaceae y Cyperaceae son las familias más representativas de las sabanas estacionales y no estacionales. El presente estudio comparte 46 especies con el estudio de Rippstein et al. (2001), quienes reportan un total de 183 especies. Los resultados del presente estudio, donde las especies más abundantes en las sabanas fueron Axonopus purpusii y Panicum trichoides, contrastan con los de Ramírez et al. (2007), en sabanas venezolanas, donde las especies más abundantes fueron Trachypogon plumosus (Humb. \& Bonpl. ex Willd.) Nees, Rhynchospora caracasana (Kunth) Boeck. La composición florística de las sabanas del presente estudio con el trabajo de Baruch (2005) muestra que solo comparte 25 especies de las 267 especies reportadas, para cuatro tipos de sabanas

Desde el punto de vista estructural, los bosques de galería no inundables registraron la mayor densidad promedio (5340 ind/ha) (Tabla 2). Las clases diamétricas mejor representadas en todos los tipos de bosque fueron las dos primeras
(0-4,9 y 5-9,9cm), excepto en los morichales, donde la clase tres $(20-49,9)$ fue la segunda mejor representada, con 115 individuos. Los individuos con DAP $\geq 50 \mathrm{~cm}$ estuvieron pobremente representados en todos los bosques y no estuvieron presentes en los morichales (Tabla 2); sin embargo, los individuos con DAP $\geq 20 \mathrm{~cm}$ representaron entre el 50 y el $96 \%$ del área basal total, en todos los tipos de bosque (Tabla 2). El área basal varió de $506,8 \mathrm{~m}^{2} /$ ha, en las matas de monte a $1347,3 \mathrm{~m}^{2} /$ ha, en el bosque de vega inundable y las especies con mayor área basal fueron Attalea butyracea (Mutis ex L.f.) Wess.Boer. $\left(580 \mathrm{~m}^{2} / \mathrm{ha}\right)$, en bosque de vega no inundable; Sloanea terniflora (Moc. \& Sesse ex DC.) Standl $\left(438,8 \mathrm{~m}^{2} / \mathrm{ha}\right)$, en bosque de vega inundable y Maquira coriaceae (H. Karst) C.C. Berg $\left(428,4 \mathrm{~m}^{2} / \mathrm{ha}\right)$, en bosque de galería inundable. Esta última especie estuvo entre los tres primeros valores de índice de valor de importancia, cobertura y abundancia relativas, en los morichales y en los bosques de galería inundables, mientras que los otros tipos de bosque no compartieron especies con mayor IVI (Tabla 3).

Son varios los factores que se deben tener en cuenta para explicar las diferencias en la composición y en la estructura, tanto de los bosques como de las sabanas del presente estudio, respecto a otros estudios realizados en la región: Factores antrópicos, dado que las sabanas estudiadas en el presente estudio han estado sujetas a procesos de disturbio, lo cual, se ve evidenciado especialmente en la estructura

Tabla 2. Densidad (ind/ha), abundancia y área basal ( $\mathrm{m}^{2} / \mathrm{ha}$ ), por categoría diamétrica de los individuos muestreados en diferentes tipos de bosque, del departamento de Casanare (Colombia).

\begin{tabular}{|c|c|c|c|c|c|c|c|c|c|c|c|c|c|c|}
\hline \multirow{3}{*}{$\begin{array}{l}\stackrel{0}{0} \\
\stackrel{0}{\circ} \\
\stackrel{0}{\circ}\end{array}$} & \multirow[b]{3}{*}{$\stackrel{\circ}{\dot{*}}$} & \multirow{3}{*}{$\begin{array}{l}\frac{\pi}{\pi} \\
\frac{\pi}{00} \\
\overline{0} \\
0\end{array}$} & \multicolumn{6}{|c|}{ Número de individuos } & \multicolumn{6}{|c|}{ Área Basal $\left(\mathrm{m}^{2} / \mathrm{ha}\right)$} \\
\hline & & & \multicolumn{6}{|c|}{ Categoría diamétrica $(\mathrm{cm})$} & \multicolumn{6}{|c|}{ Categoría diamétrica $(\mathrm{cm})$} \\
\hline & & & $0-4,9$ & $5-9,9$ & $\begin{array}{l}10- \\
19,9\end{array}$ & $\begin{array}{l}20- \\
49,9\end{array}$ & $\geq 50$ & Total & $0-4,9$ & $5-9,9$ & $\begin{array}{l}10- \\
19,9 \\
\end{array}$ & $\begin{array}{l}20- \\
49,9 \\
\end{array}$ & $\geq 50$ & Total \\
\hline \multirow{2}{*}{ BGI } & 2 & 4890 & 207 & 153 & 95 & 34 & & 489 & 17,3 & 51,2 & 157,2 & 225,7 & & 451,4 \\
\hline & 6 & 4300 & 158 & 158 & 83 & 28 & 3 & 430 & 13,1 & 55,1 & 114,9 & 189,1 & 300,0 & 672,3 \\
\hline \multirow{2}{*}{ BGNI } & 9 & 6570 & 262 & 311 & 31 & 53 & & 657 & 23,1 & 87,1 & 50,1 & 404,2 & & 564,5 \\
\hline & 10 & 4110 & 188 & 125 & 51 & 45 & 2 & 411 & 13,8 & 38,7 & 72,9 & 326,4 & 39,3 & 491,1 \\
\hline BVI & 12 & 2560 & 82 & 78 & 67 & 23 & 6 & 256 & 7,2 & 28,0 & 94,9 & 161,0 & 742,2 & 1033,3 \\
\hline \multirow{2}{*}{ BVNI } & 13 & 1450 & 49 & 40 & 15 & 32 & 9 & 145 & 3,8 & 14,5 & 22,4 & 374,6 & 363,4 & 778,7 \\
\hline & 14 & 1500 & 32 & 46 & 55 & 15 & 2 & 150 & 3,4 & 17,1 & 82,5 & 131,4 & 61,5 & 295,9 \\
\hline MM & 5 & 2050 & 89 & 64 & 22 & 25 & 5 & 205 & 7,7 & 19,5 & 35,4 & 194,7 & 249,5 & 506,8 \\
\hline \multirow{2}{*}{ Mori } & 3 & 3240 & 170 & 58 & 30 & 66 & & 324 & 15,0 & 18,4 & 46,4 & 502,6 & & 582,4 \\
\hline & 7 & 1500 & 31 & 34 & 36 & 49 & & 150 & 2,1 & 15,2 & 58,1 & 337,8 & & 413,3 \\
\hline
\end{tabular}

BGI: bosque de galería inundable, BGNI: bosque de galería no inundable, BVNI: bosque de vega no inundable, MM: mata de monte, Mori: Morichal. 
Tabla 3. Índice de valor de importancia (\%) de las tres especies vegetales más dominantes en diferentes tipos de bosque, del departamento de Casanare (Colombia). El IVI fue obtenido sumando la cobertura relativa (área basal) y la abundancia relativa.

\begin{tabular}{|c|c|c|c|c|c|c|c|c|c|c|}
\hline Especie & BGI-1 & BGI-2 & $\begin{array}{c}\text { BGNI- } \\
1\end{array}$ & $\begin{array}{l}\text { BGNI- } \\
2\end{array}$ & BVI & $\begin{array}{c}\text { BVNI- } \\
1\end{array}$ & $\begin{array}{l}\text { BVNI- } \\
2\end{array}$ & MM & Mori-1 & Mori-2 \\
\hline Protium llanorum Cuatrec. & 35,8 & 57,5 & & & & & & & & \\
\hline $\begin{array}{l}\text { Calophyllum brasiliense } \\
\text { Cambess. }\end{array}$ & 29,9 & 19,3 & & & & & & & & \\
\hline $\begin{array}{l}\text { Maquira coriacea } \\
\text { (H. Karst.) C.C. Berg }\end{array}$ & 28,2 & 57,5 & & & & & & & 44,9 & 105,6 \\
\hline Attalea insignis (Mart.) Drude & & & 109,3 & 71,1 & & & & & & \\
\hline Bactris major Jacq. & & & 12,5 & & & & & & & \\
\hline $\begin{array}{l}\text { Himatanthus articulatus } \\
\text { (Vahl) Woodson }\end{array}$ & & & 12,3 & & & & & & & \\
\hline $\begin{array}{l}\text { Warszewiczia coccinea } \\
\text { (Vahl) Klotzsch }\end{array}$ & & & & 20,2 & & & & & & \\
\hline $\begin{array}{l}\text { Licania subarachnophylla } \\
\text { Cuatrec. }\end{array}$ & & & & 12,6 & & & & & & \\
\hline $\begin{array}{l}\text { Sloanea terniflora } \\
\text { (Sessé \& Moç. ex DC). Standl. }\end{array}$ & & & & & 44,8 & & & & & \\
\hline Mouriri guianensis Aubl. & & & & & 27,4 & & & & & \\
\hline Byrsonima japurensis A. Juss. & & & & & 26,6 & & & & & \\
\hline $\begin{array}{l}\text { Trichostigma octandrum } \\
\text { (L.) H.Walter }\end{array}$ & & & & & & 100,0 & & & & \\
\hline $\begin{array}{l}\text { Attalea butyracea (Mutis ex } \\
\text { L.f.) Wess.Boer. }\end{array}$ & & & & & & 91,5 & 60,7 & & & \\
\hline Ficus insipida Willd. & & & & & & 34,7 & & & & \\
\hline $\begin{array}{l}\text { Trichanthera gigantea } \\
\text { (Bonpl.) Nees }\end{array}$ & & & & & & & 35,2 & & & \\
\hline Tacarcuna amanoifolia Huft & & & & & & & 32,2 & & & \\
\hline Ocotea floribunda (Sw.) Mez & & & & & & & & 50,9 & & \\
\hline Ocotea longifolia Kunth & & & & & & & & 35,2 & & \\
\hline Ficus sp1 & & & & & & & & 19,2 & & \\
\hline $\begin{array}{l}\text { Mauritia flexuosa L. f. var. } \\
\text { venezuelana Steyerm }\end{array}$ & & & & & & & & & 50,9 & 23,8 \\
\hline $\begin{array}{l}\text { Parahancornia oblonga } \\
\text { (Benth. ex Müll. Arg.) Monach. }\end{array}$ & & & & & & & & & 33,0 & \\
\hline $\begin{array}{l}\text { Virola surinamensis } \\
\text { (Rol. ex Rottb.) Warb. }\end{array}$ & & & & & & & & & & 15,6 \\
\hline
\end{tabular}

BGI: bosque de galería inundable; BGNI: bosque de galería no inundable; BVNI: bosque de vega no inundable; MM: mata de monte; Mori: Morichal. 
de las coberturas de bosque; factores relacionados con las metodologías o en las estaciones del año fueron las que se hicieron en los muestreos; factores, como la alta heterogeneidad ambiental, que muestran las sabanas tropicales latinoamericanas. Al respecto, Baruch (2005) señala, en el caso de las sabanas venezolanas, que muestran una alta heterogeneidad florística y estructural y relaciones complejas con su ambiente local. La variabilidad espacial y las interacciones entre la morfología del plano de inundación, el régimen hidrológico y las características del suelo son fundamentales para estructurar la comunidad de bosques ribereños (Veneklaas et al. 2005). Así, los bosques de Veneklaas et al. (2005) corresponden a una altillanura plana al sur del río Meta, caracterizada por una vegetación de sabana abierta y algunos bajos y vías de drenaje, mientras que los de Cabrera-Amaya \& Rivera-Díaz (2016) corresponden a las denominadas llanuras aluviales de desborde y llanuras eólicas (Mora-Fernández et al. 2013) y a sabana hiperestacional mal drenada al norte del río Meta (Serna-Isaza et al. 2001). Finalmente, los contrastes en los resultados, se pueden deber a diferencias biogeográficas o bióticas, que se constituyen, según Ricklefs (2004), en determinantes regionales y locales de la diversidad; al respecto, Correa-Gómez \& Stevenson (2010) sugieren dominancia local en la región.

Este estudio contribuye con el conocimiento de la flora del departamento de Casanare, de la cual, se reportan 146 familias, 659 géneros y 1479 especies (Córdoba et al. 2011), sumando 83 especies, 20 géneros y 6 familias, solo en el municipio de Orocué. Los bosques ribereños estudiados muestran una marcada diferencia en la composición y en la estructura con otros bosques similares en la región, mientras que las sabanas son más similares. Estos resultados sugieren una alta heterogeneidad ambiental, lo que resalta la necesidad de continuar ampliando el conocimiento de la vegetación de la Orinoquía colombiana, especialmente, si se tiene en cuenta que solo el $2 \%$ del departamento tiene alguna figura de protección y, de la cual, el $70 \%$ tiene algún tipo de intervención (Córdoba et al. 2011).

Agradecimientos: Agradecemos a los propietarios de las fincas, por permitirnos realizar el estudio. A Leticia Camacho de Vidales y Alexander Bernal, por su apoyo logístico. A Fernando Gaitán, Gilberto Mazonas y Franklin Cuevas, por su apoyo en campo. Este estudio fue financiado por la Universidad de Ciencias Aplicadas y Ambientales U.D.C.A. Conflicto de intereses: El manuscrito fue preparado y revisado con la participación de todos los autores, quienes declaramos que no existe conflicto de intereses que ponga en riesgo la validez de los resultados presentados.

\section{BIBLIOGRAFÍA}

1. ANGIOSPERM PHYLOGENY GROUP -APG-. 2009. An update of the Angiosperm Phylogeny Group classification for the orders and families of flowering plants: APG III. Botanical J. Linnean Society (Inglaterra). 161(2):105-121.

2. AGUIRRE, N. 1999. Casanare: características geográficas. Departamento Administrativo Nacional de Estadísticas - Instituto Geográfico Agustín Codazzi. (Bogotá). 356p.

3. ARISTEGUIETA, L. 1968. Consideraciones sobre la flora de los morichales llaneros al norte del Orinoco. Acta. Bot. Venez. 3(1/4):1-22.

4. BARBOSA, C. 1992. Contribución al conocimiento de la flórula del Parque Nacional Natural El Tuparro. Libro No. 3. Serie de publicaciones especiales del INDERENA-Biblioteca Andrés Posada Arango (Bogotá) 271p.

5. BARUCH, Z. 2005. Vegetation-environment relationships and classification of the seasonal savannas in Venezuela. Flora (Alemania). 200(1):49-64.

6. CABRERA-AMAYA, D.; RIVERA-DÍAZ, O. 2016. Composición florística y estructura de los bosques ribereños de la cuenca baja del río Pauto, Casanare, Colombia. Caldasia (Colombia). 38(1):53-85.

7. CÁRDENAS, J. 2007. Flora del Escudo Guayanés en Inírida (Guainía, Colombia). Instituto Amazónico de Investigaciones Científicas - SINCHI (Bogotá). 186p.

8. CASTRO-LIMA, F. 2010. Avance del conocimiento de la flora del Andén Orinoqués en el departamento del Vichada, Colombia. Orinoquía (Colombia). 14(1):5867.

9. CÓRDOBA-SÁNCHEZ, M.; MIRANDA-CORTÉS, L.; ÁVILA-AVILÁN, R.; PÉREZ-ROJAS, C. 2011. Flora de Casanare. En: Usma J, Trujillo F, (eds). Biodiversidad del Casanare: Ecosistemas Estratégicos del Departamento. Gobernación de Casanare - WWF Colombia (Bogotá). p.82-101.

10. CORREA-GÓMEZ, D.; STEVENSON, P. 2010. Estructura y diversidad de bosques de galería en una sabana estacional de los llanos orientales colombianos (Reserva Tomo Grande, Vichada). Orinoquía (Colombia). 14(1):31-48. 
11. CORREA, H.; RUIZ, S.; ARÉVALO, L. (eds). 2005. Plan de acción en biodiversidad de la cuenca del Orinoco - Colombia / 2005 - 2015 - Propuesta Técnica. Corporinoquia, Cormacarena, I.A.v.H, Unitrópico, Fundación Omacha, Fundación Horizonte Verde, Universidad Javeriana, Unillanos, WWF - Colombia, GTZ - Colombia. (Bogotá). 273p.

12. CORTÉS, R.; FRANCO, O.; RANGEL, O. 1998. La flora vascular de la Sierra de Chiribiquete, Colombia. Caldasia. 20(2):103-141.

13. DELASCIO, F. 1990. Contribución al conocimiento florístico de los morichales del estado Guarico, Venezuela: Morichal Redondo, Hato Becerra. Acta Bot. Venez. 16(1):27-34.

14. DOMÍNGUEZ, C. 1998. La gran cuenca del Orinoco. En: Fajardo, M. Colombia Orinoco. Fondo FEN (Bogotá). p.39-67.

15. DUNO DE STEFANO, R.; AYMARD, G.; HUBER, O. (eds). 2007. Catálogo ilustrado y anotado de las plantas vasculares de los Llanos de Venezuela. FUDENA - Fundación Empresas Polar - FIBV. (CARACAS). 738p.

16. ETTER, A. 2001. El Escudo de Guayana. En: Etter, A. (ed). Puinawai y Nukak. Caracterización Ecológica General de dos Reservas Nacionales Naturales de la Amazonía Colombiana. Instituto de Estudios Ambientales para el Desarrollo - IDEADE. Serie Investigación 2. (Bogotá). p.31-42.

17. FERNÁNDEZ, A.; GONTO, R.; RIAL, A.; ROSALES, J.; SALAMANCA, B.; CÓRDOBA, M.; CASTRO, F.; ALCÁZAR, C.; GARCÍA, H.; ARIZA, A. 2010. Flora y vegetación de la cuenca del río Orinoco. En: Lasso, C.A.; Usma, J.S.; Trujillo, F.; Rial, A. (eds). Biodiversidad de la cuenca del Orinoco: bases científicas para la identificación de áreas prioritarias para la conservación y uso sostenible de la biodiversidad. Instituto de Investigación de Recursos Biológicos Alexander von Humboldt, WWF Colombia, Fundación Omacha, Fundación La Salle e Instituto de Estudios de la Orinoquía de la Universidad Nacional de Colombia. (Bogotá). p.126-195.

18. GRACE, J.; JOSE, J.; MEIR, P.; MIRANDA, H.; MONTES, R. 2006. Productivity and carbon fluxes of tropical savannas. J. Biogeogr. (Internacional). 33:387-400.
19. HOLDRIDGE, L. 1947. Determination of World Plant Formations from Simple Climatic Data. Science (USA) 105(2727):367-368.

20. LASSO, C.; RIAL, A.; GONZALEZ-B, V. (eds). 2013. Morichales y cananguchales de la Orinoquia y Amazonia: Colombia-Venezuela. Parte I/Serie Recursos Hidrobiológicos y Pesqueros Continentales de Colombia, VII. Bogotá: Instituto de Investigación de Recursos Biológicos Alexander von Humboldt (Bogotá). 339p.

21. MENDOZA, H. 2011. Vegetación. En: Villareal-Leal, H.; Maldonado-Ocampo, J. (eds). Caracterización biológica del Parque Nacional Natural El Tuparro (Sector noreste), Vichada, Colombia. Instituto de Investigación de Recursos Biológicos Alexander von Humboldt. (Bogotá). p.53-84.

22. MORA-FERNÁNDEZ, C.; PEÑUELA-RECIO, L. 2013. La salud ecosistémica en las sabanas inundables orinocenses. En: Mora-Fernández, C.; Peñuela-Recio, L. (eds). Salud ecosistémica de las sabanas inundables asociadas a la cuenca del río Pauto, Casanare, Colombia. Yoluka PNG, Fundación de Investigación en Biodiversidad y conservación, Fundación Horizonte Verde y Ecopetrol S.A. (Bogotá). p.34-41.

23. MORA-FERNÁNDEZ, C.; PENUELA-RECIO, L.; CASTRO-LIMA, F. 2015. Estado do conhecimento sobre os ecossistemas das savanas inundadas no Orinoquia Colombiana. Orinoquia. 19:253-271.

24. PARRA C. 2006. Estudio general de la vegetación nativa de Puerto Carreño (Vichada, Colombia). Caldasia. 28(2):165-177.

25. RAMIA, M. 1967. Tipos de sabanas en los llanos de Venezuela. Bol. Soc. Venez Cienc Nat. 27:264-288.

26. RAMÍREZ, N.; DEZZEO, N.; CHACÓN, N. 2007. Floristic composition, plant species abundante, and soil propierties of montane savannas in the Gran Sabana, Venezuela. Flora (Alemania). 202:316-327.

27. RAUSCH, J. 2009. Petroleum and the transformation of the Llanos Frontier in Colombia: 1980 to the present. Lat. Am. (Internacional). 53:113-136.

28. RICKLEFS, R. 2004. A comprehensive framework for global patterns in biodiversity. Ecol. Lett. (Internacional). 7:1-15. 
29. RIPPSTEIN, G.; ESCOBAR, E.; TOLEDO, J.; FISHER, M.; MESA, E. 2001. Caracterización de comunidades vegetales de la altillanura en el Centro de Investigación Agropecuaria Carimagua, en Meta, Colombia. En: Rippstein, G.; Escobar, G.; Motta, F. (eds). Agroecología y biodiversidad de las sabanas de los llanos orientales de Colombia. Centro Internacional de Agricultura Tropical. (Cali). p.22-45.

30. ROMERO-RUIZ, M.; GALINDO, G.; OTERO, J.; ARMENTERAS, D. 2004. Ecosistemas de la cuenca del Orinoco Colombiano. Bogotá: Instituto de Investigaciones en Recursos Biológicos "Alexander von Humboldt" e Instituto Geográfico Agustin Codazzi e IGAC. (Bogotá). 189p.

31. RUDAS, A.; PRIETO, A.; RANGEL, O. 2002. Principales tipos de vegetación de "La Ceiba" (Guainía), Guayana colombiana. Caldasia. 24(2):343-365.

32. SARMIENTO, G.; VERA, M. 1979. Composición, estructura, biomasa y producción primaria de diferentes sabanas de los Llanos Occidentales de Venezuela. Bol. Soc. Venez. Ci. Nat. 34(136):5-42.

33. SERNA-ISAZA, R.; RIPPSTEIN, G.; GROLLIER, C.; MESA, E. 2001. Biodiversidad de la vegetación de sabana en la altillanura plana y la serranía de los lla- nos orientales. En: Rippstein, G.; Escobar, G.; Motta, F. (eds). Agroecología y biodiversidad de las sabanas de los llanos orientales de Colombia. Centro Internacional de Agricultura Tropical. (Cali). p.46-63.

34. TEJOS, M. 2000. Caracterización y manejo de pastos nativos de sabanas inundables. En: Tejos, M.; Zambrano, C.; Mancilla, L.; García, W. (eds). VI Seminario de Manejo y Utilización de Pastos y Forrajes en Sistemas de Producción Animal. Unellez. (Barinas). p.16-25.

35. USMA, J.S.; TRUJILLO, F. 2011. Biodiversidad del Casanare: Ecosistemas Estratégicos del Departamento. (Eds). Gobernación de Casanare - WWF Colombia. Bogotá D.C. 286p.

36. VENEKLAAS, E.; FAJARDO, A.; OBREGON, S.; LOZANO, J. 2005. Gallery forest types and their environmental correlates in a Colombian savanna landscape. Ecography (Internacional). 28:236-252.

37. VINCELLI, P. 1981. Estudio de la Vegetación del Territorio Faunístico El Tuparro. Cespedesia. (Colombia). 10(37-38):7-54.

Recibido: Abril 20 de 2017

Aceptado: Junio 1 de 2018

Cómo citar:

Romero-Duque, L.P.; Castro-Lima, F.; Rentería-Mosquera, A. 2018. Contribución al conocimiento de la vegetación de las Sabanas de Casanare (Colombia). Rev. U.D.C.A Act. \& Div. Cient. 21(1): 197-205. 